\title{
Benign chondroid syringoma of foot: a clinical dilemma
}

\begin{abstract}
Chondroid syringoma is a rare skin appendageal tumor which is most commonly encountered in the head and neck region. We herein report an unusual case of a benign chondroid syringoma of the left foot in a 40-year-old female which posed as a diagnostic conundrum. The approach to such a case, differential diagnosis and the management of this rare entity is also presented.
\end{abstract}

Keywords: benign, chondroid syringoma, cytology, foot
Volume II Issue 2 - 2020

\author{
Sonam Sharma,' Sansar Chand Sharma ${ }^{2}$ \\ 'Department of Pathology, Kalpana Chawla Government \\ Medical College, Karnal, India \\ ${ }^{2}$ Department of Orthopaedics, Faculty of Medicine and Health \\ Sciences, SGT University, Gurgaon, India
}

Correspondence: Sonam Sharma, Department of Pathology, Kalpana Chawla Government Medical College, Karnal, Haryana, India,Tel 999984I393,Email drsonamsharma@gmail.com

Received: January 31, 2020 | Published: March II, 2020

\section{Introduction}

Chondroid syringoma is an uncommon skin adnexal tumor which arises from both the secretory as well as the ductal elements of the sweat gland. Among all the primary tumors of the skin, the reported incidence of chondroid syringoma is low, ranging between $0.01 \%$ to $0.098 \%$ and it exists in two forms i.e. benign and malignant. ${ }^{1,2}$ The benign type has a predilection for the head and neck region of the middle-aged men whereas, the malignant type is more commonly encountered at the extremities of the females and is characterized by rapid growth, local invasion, and distant metastasis. ${ }^{3}$ We describe a rare case of a benign chondroid syringoma with an atypical location on the left foot of a 40-year-old female and also highlight the pivotal role of cytology in such an unusual setting.

\section{Case report}

A 40-year-old illiterate female from a rural background presented to the orthopaedic outpatient department with a swelling over the lateral border of the left foot in the midfoot area since last 15 years. She revealed that the swelling was initially pea sized but gradually over a period of last 6 months it has increased in its size. History of any trauma, pain, itching or infection of this area was noncontributory. Her personal, past, family and medical history was insignificant. Local examination of the left foot revealed a non-tender, mobile, firm swelling that measured $1 \times 0.8 \mathrm{~cm}$ in size. The overlying skin was normal (Figure 1). Her hematological investigations revealed microcytic hypochromic anemia with hemoglobin level of $10.5 \mathrm{gm} / \mathrm{dl}$, total leucocyte count of $10,500 / \mathrm{mm}^{3}$ with differential being of $75 \%$ neutrophils and a platelet count of $2,00,000 / \mathrm{mm}^{3}$. The erythrocyte sedimentation rate was $18 \mathrm{cumm} / \mathrm{hr}$. Renal as well as liver function tests were normal. Urine and blood cultures were negative. X-ray of the left foot showed a well-defined soft tissue mass in the midfoot area without any bony involvement (Figure 2). Based on the history of the patient and the clinical findings, a provisional diagnosis of a benign mesenchymal lesion was made. To arrive at a conclusive diagnosis, Fine Needle Aspiration Cytology (FNAC) was performed. Two passes at two different sites of the lesion were done. Thick mucoid material was aspirated in both the attempts. This material was pushed onto the clean glass slides and the smears were drawn. The smears prepared, were stained with May-Grunwald-Giemsa (MGG) stain followed by microscopic examination. FNAC smears were cellular on microscopy and revealed distinct epithelial and mesenchymal components. The epithelial cells were round and had moderate amount of cytoplasm with a central round to oval bland nucleus having finely dispersed chromatin. The myoepithelial cells were larger and had moderate to abundant amount of pale blue cytoplasm and an eccentric round nucleus giving the cell a plasmacytoid appearance. Both these cell types were embedded within the chondromyxoid fibrillary stroma. There was no evidence of any nuclear atypia, prominent nucleoli or tumor diathesis (Figure 3). Based on these cytomorphological findings, a diagnosis of a benign chondroid syringoma of the left foot was rendered and excision of the mass was advised. The mass was resected along with a margin of the normal surrounding tissue and the specimen was sent for histopathological examination. Grossly, the mass was partly skin covered, grey white to grey brown in color, wellcircumscribed, firm in consistency and measured around $1 \times 1 \times 0.5 \mathrm{~cm}$ in size. On cut section, it was greyish white and had focal cystic spaces which were filled with mucoid material. Formalin fixed paraffin embedded routine Hematoxylin and Eosin stained microsections revealed a well-circumscribed tumor comprising of proliferating epithelial cells, interspersed in chondromyxoid and fibrocollagenous stroma. Numerous nests of polygonal cells and interconnecting tubuloalveolar structures lined by two layers of cuboidal epithelial cells were seen. Ductal structures lined by a single layer of epithelial cells were also apparent. Cellular pleomorphism was absent (Figure 4). These histopathological findings pointed towards the final diagnosis of a benign chondroid syringoma (eccrine type) of the left foot. Immunohistochemistry (IHC) was also performed, which revealed a strong positivity of inner epithelial layer for cytokeratin (CK) 7 as well as epithelial membrane antigen (EMA) and the outer myoepithelial layer for vimentin, S100 and p63. A negative immunoexpression of tumor cells for p15, p16 and desmin was seen. The post-operative period of the patient was uneventful. There was no evidence of recurrence of the lesion during her 2 year follow-up period. 


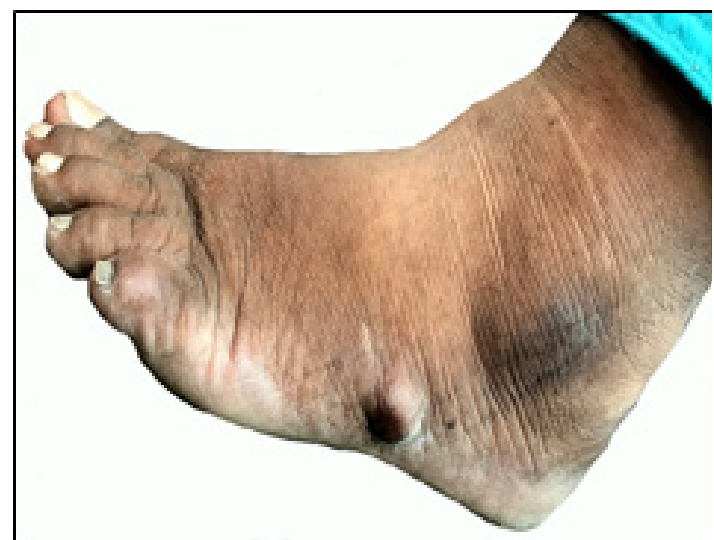

Figure I A nodular swelling of the left foot on clinical inspection.

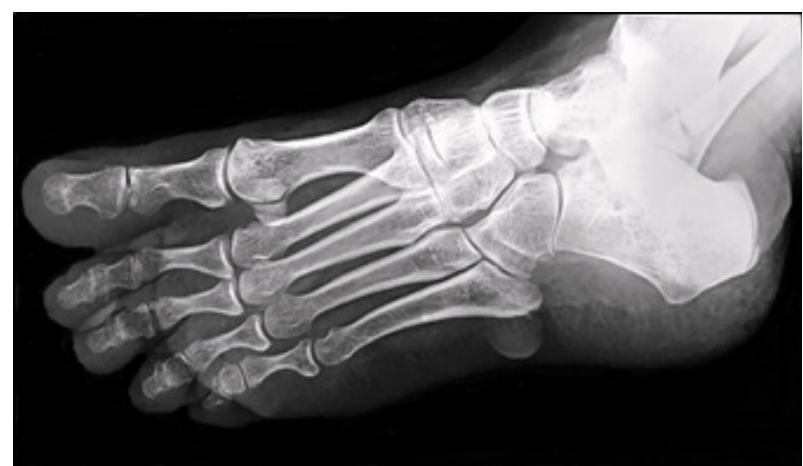

Figure 2 X-ray of the left foot (oblique view) exhibiting a well-defined radiopaque lesion in the midfoot area.

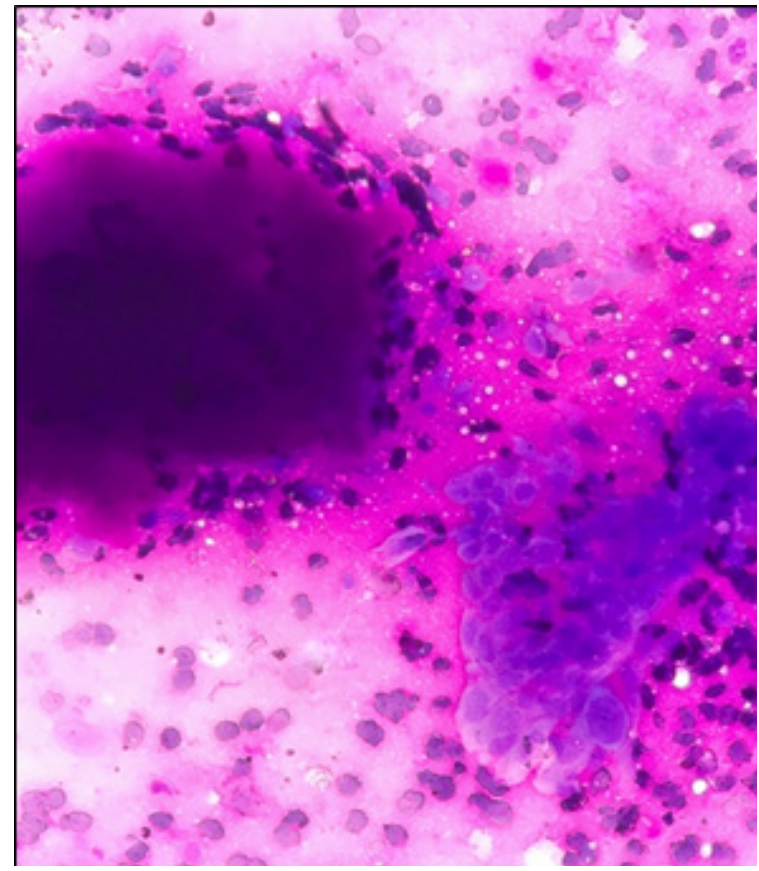

Figure 3 Metachromatic fibrillary stroma with spindled myoepithelial cells (MGG, x400).

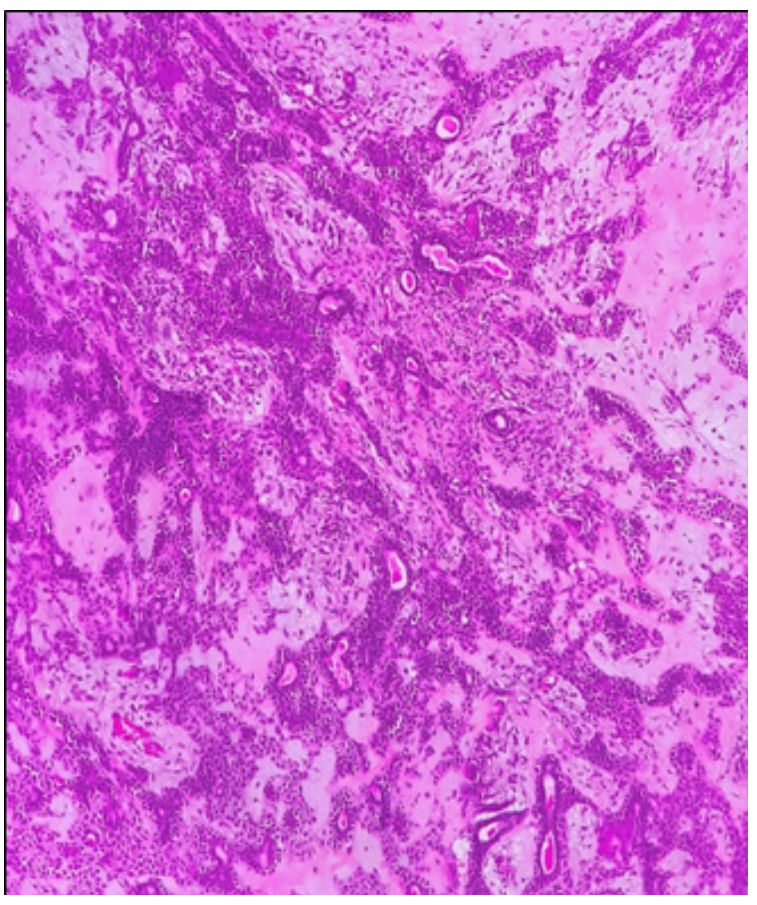

Figure 4 Photomicrograph exhibiting the epithelial cells with myxoid connective tissue stroma $(\mathrm{H} \& \mathrm{E}, \mathrm{xI00})$.

\section{Discussion}

Chondroid syringoma, also known as a cutaneous mixed tumor is a rare skin appendageal neoplasm which can be classified into apocrine or eccrine types, based on the histopathological appearance of the sweat gland lumina within the tumor. ${ }^{4}$ The term, mixed tumor, was coined by Virchow and Minssen for tumors with microscopic features that indicate both epithelial and mesenchymal origin. ${ }^{5}$ Since then this term has been used to describe the cutaneous tumors as well as tumors of salivary glands, uterus, lung, pancreas and the kidney. ${ }^{6}$ In 1859 , Billroth, originally defined the mixed tumor of the skin as an entity having the same histopathological features of the mixed tumors of the salivary glands i.e. pleomorphic adenoma. ${ }^{7}$ However, it was in 1961 that the term 'chondroid syringoma' was first introduced by Hirsch \& Helwig $^{8}$ to describe this neoplasm because of the presence of sweat gland elements set in a cartilaginous stroma. They also proposed the histological diagnostic criteria of this uncommon skin adnexal tumor.

The diagnosis of chondroid syringoma in routine clinical practice is difficult owing to its rarity as well as silent clinical presentations. Nevertheless, there are certain clinical differences that can assist in differentiating its benign and malignant forms. The benign chondroid syringoma generally arises in the head and neck region, but most commonly affects the nose, cheek and upper lip. ${ }^{9}$ Less frequently, this tumor can develop on the scalp, eyelid, hand, foot, forehead, axilla, abdomen, penis, vulva, and scrotum. ${ }^{10}$ It is mainly encountered in middle-aged males. The gross appearance of the tumor is typically a slow-growing, solitary, solid, painless, non-ulcerating, wellcircumscribed subcutaneous, or intracutaneous nodule ranging from 0.5 to $3.0 \mathrm{~cm}$ in size. The malignant variant tends to affect the lower extremities and trunk. There is a predilection towards females however, there does not seem to be any correlation with the age. It shows rapid 
growth or ulceration and the size of the tumor is usually greater than $3.0 \mathrm{~cm} .{ }^{3}$ However, in most of the cases, chondroid syringoma is not clinically distinctive and thus is often overlooked or misdiagnosed with other types of skin lesions, such as dermoid or sebaceous cysts, neurofibroma, dermatofibroma, basal cell carcinoma, pilomatrixoma, histiocytoma, and seborrheic keratosis. ${ }^{5}$ Radiological findings of chondroid syringoma are also non-specific. However, modalities like Magnetic Resonance Imaging can accurately depict the anatomical extent and identify tissue of origin, depth of invasion and relation to the adjacent structures, such as muscles and bones. ${ }^{11}$ Therefore, due to the paucity of specific diagnostic clinical and radiological features, the pathological evaluation is imperative for a definite diagnosis of chondroid syringoma especially for those occurring at atypical sites. ${ }^{12}$

Many researchers have documented that FNAC has been a boon for an early diagnosis of cutaneous or subcutaneous nodules as it is not only a rapid, cost-effective diagnostic tool but it is also an excellent non-invasive method because in the presence of characteristic cytomorphology, it obviates the need for more invasive procedures. Ancillary tests like immunocytochemistry, cell block formation, liquid based cytology followed by histopathological examination and IHC have also broadened the utilization of FNAC for the definite diagnosis of many tumors including those arising from skin adnexa. ${ }^{13-25}$ In chondroid syringoma, the fine needle aspirate is usually thick and has mucoid like consistency. A cytological criteria has also been documented for its definitive diagnosis which comprises of a prominent cellular component of epithelial and myoepithelial cells embedded in a fibrillary metachromatic chondromyxoid background. ${ }^{26}$ However, epithelial cells with marked nuclear pleomorphism and prominent nucleoli against a myxoid background and few chondroid foci helps in differentiating malignant and benign forms. ${ }^{27}$ Nevertheless, the histopathological examination of tissue obtained by excisional skin biopsy is essential for the final diagnosis of chondroid syringoma. Histologically, it consists of mixed epithelial and mesenchymal elements. The epithelial cells are arranged in cords, ducts, or tubules with a myoepithelial cell layer. The stromal component is most often myxoid-cartilaginous (chondromyxoid), but may also be hyalinized/fibrous, fatty, osteoid, or may even be minimal or absent. The stromal cells show myoepithelial differentiation. ${ }^{28}$ Two histological variants of this tumor are described, the eccrine type with smaller lumens, lined by a single row of cuboidal epithelial cells and the apocrine variant with tubular and cystic branching lumina, lined by two rows of epithelial cells. ${ }^{5}$ Some tumors may exhibit both the apocrine and eccrine features. Although, the malignant chondroid syringoma typically arises de novo and not from a pre-existing benign tumor, there are certain histological features indicating malignancy which include cytologic atypia, infiltrative margins, tumor necrosis, numerous mitosis, excessive mucoid matrix, poorly differentiated chondroid components, satellite tumor nodules and involvement of deeper structures. ${ }^{3,29}$ Few authors have also documented that the presence of a large amount of mucoid matrix and poor chondroid differentiation in a malignant chondroid syringoma are the true determinants of its metastatic potential. ${ }^{30}$ Therefore, examination of the entire specimen is essential to exclude any area of malignant transformation.

Sometimes it is difficult to differentiate whether the chondroid syringoma is either an eccrine/apocrine or benign/malignant type. It might also be difficult to distinguish this neoplasm from other metastatic skin lesions, specific dermal structures and primary skin tumors; as these types of lesion frequently share similar morphological patterns on histopathology. In such cases, immunohistochemical markers play a significant role in differentiating these entities. The inner cell layer of this tumor expresses epithelial markers, including CK, EMA, and carcinoembryogenic antigen (CEA). The outer cell layer is negative for CK, EMA, and CEA, but stain positive with mesenchymal markers, including S-100, vimentin, neurone-specific enolase, and less commonly by glial fibrillary acid protein, smooth muscle actin, calponin and p63. Desmin is usually negative. p15, is considered an apocrine marker and p16 is positive in malignant chondroid syringoma. Both these markers were negative in our case suggesting an eccrine differentiation and benign form of chondroid syringoma. $^{31}$

Therapeutically, the treatment of choice for chondroid syringoma is wide surgical excision including a margin of the surrounding normal tissue. Other surgical modalities for its treatment are electrodesiccation, dermabrasion, and vaporization with argon or $\mathrm{CO}_{2}$ lasers. ${ }^{5} \mathrm{An}$ inadequate resection of the tumor leading to its local recurrence and the potential risk of malignant transformation are one of the major post-operative concerns of chondroid syringoma. Therefore, the excision techniques that do not allow pathological evaluation of the entire specimen should be avoided. ${ }^{32}$ Nevertheless, the recurrent lesions can be surgically re-excised and an excision biopsy still remains the most reliable method for chondroid syringoma for preventing its recurrence and in determining its malignant potential. On the other hand, the malignant chondroid syringoma is usually associated with metastasis to the regional lymph nodes, lung and bones. Few authors have recommended that regional lymph node resection can be done in the presence of clinically suspicious or palpable lymph node metastasis and also an aggressive surgery and adjuvant radiotherapy, with or without chemotherapy as an initial treatment option can be helpful for treating malignant tumors. ${ }^{33}$ Therefore, periodic long term follow-up pattern is mandatory as the rate of recurrence and malignant changes even after many years of surgical excision is quite high in such patients.

The present case is unique because of the location of this benign tumor which is typically seen in the head and neck region. According to the pertinent world literature, a very few cases of benign chondroid syringoma arising from the foot have been documented till date. ${ }^{34-}$ ${ }^{36}$ It was clinically misdiagnosed as a benign mesenchymal lesion of the left foot and the radiology was inconclusive. However, the FNAC played an important role in clinching the pre-operative diagnosis this rare tumor. Wide local excision was done and the histopathological evaluation of resected specimen was consistent with the cytological diagnosis of a benign chondroid syringoma of the left foot. No recurrences or any fresh complaints have been reported so far by the patient even after 2 year of follow-up.

\section{Conclusion}

Chondroid syringoma of the foot is a rare neoplasm which should always be kept in mind while dealing with foot swellings. A high index of suspicion and cytological investigation play a pivotal role in clinching an early definite diagnosis. However, the histopathological examination of the resected tumor obtained after complete wide surgical excision is essential. Tumor recurrence as well as malignant changes within the tumor itself is a concern which makes a long-term follow-up period necessary in all such cases.

\section{Acknowledgments}

None. 


\section{Conflicts of interest}

No financial interest or any conflict of interest exists.

\section{References}

1. Mebazaa A, Trabelsi S, Denguezli M, et al. Chondroid syringoma of the arm: An unusual localization. Dermatol Online J. 2006;12(1):14.

2. Sivamani RK, Wadhera A, Craig E. Chondroid syringoma: case report and review of the literature. Dermatol Online J. 2006;12(5):8.

3. Sungur N, Uysal A, Gümüş M, et al. An unusual chondroid syringoma. Dermatol Surg. 2003;29(9):977-979.

4. Headington JT. Mixed tumors of skin: Eccrine and apocrine types. Arch Dermatol. 1961;84:989-996.

5. Yavuzer R, Basterzi Y, Sari A, et al. Chondroid syringoma: a diagnosis more frequent than expected. Dermatol Surg. 2003;29(2):179-181.

6. Lakshmi T, Gnaneshwar AR. Multiple chondroid syringoma. Indian J Dermatol Venereol Leprol. 1988;64(2):83-84.

7. Sheikh SS, Pennanen M, Montgomery E. Benign chondroid syringoma: report of a case clinically mimicking a malignant neoplasm. J Surg Oncol. 2000;73(4):228-230.

8. Hirsch P, Helwig EB. Chondroid syringoma. Mixed tumor of skin, salivary gland type. Arch Dermatol.1961;84:835-847.

9. Storm CA, Seykora JT. Cutaneous adnexal neoplasms. Am J Clin Pathol. 2002;118 Suppl:S33-S49.

10. Khan K, Dinesh A, Landa M, et al. A rare forehead mass: the chondroid syringoma. Cureus. 2019;11(9):e5763.

11. Goldberg SH, Cantore WA. Tumors of the orbit. Curr Opin Ophthalmol.1997;8(5):51-56.

12. Sirivella S, Gielchinsky I. Chondroid syringoma: a rare tumor of the chest wall. Ann Thorac Surg. 2010;89(3):983-985.

13. Mannan R, Piplani S, Sharma S, et al. Cytological correlation of spectrum of head and neck lesions with epidemiological and diagnostic parameters. Indian J Pathol Oncol. 2017;4(1):92-97.

14. Sharma S, Mannan R, Bhasin T. Cytological diagnosis of deep-seated cellular hemangioma of the parotid gland by using cell button technique. J Cytol. 2016;33(3):174-176.

15. Rajpoot J, Sharma S, Arora R. Recurrent cutaneous leiomyosarcoma of the anterior abdominal wall: a rare tumor at an unusual site. Int $J$ Res Dermatol. 2017;3(1):151-154.

16. Sharma S, Sonali. Cutaneous metastasis from an asymptomatic breast cancer masquerading as an infected sebaceous cyst. Madridge J Dermtol Res. 2018;3(1):46-50.

17. Singh P, Mittal MK, Sharma S. Thyrolipoma: a rare thyroid gland entity. Nepalese Journal of Radiology. 2019;9(13):24-29.

18. Bhasin T, Mannan R, Manjari M, et al. Reproducibility of 'the Bethesda system for reporting thyroid cytopathology': A multicenter study with review of the literature. J Clin Diagn Res. 2013;7(6):1051-1054.
19. Sharma S, Mandal D, Yadav AK, et al. Primary thyroid lymphoma: a comprehensive summary of two cases. Int J Sci Rep. 2015;1(3):177-180.

20. Kaur J, Sharma S, Bhasin TS, et al. A rare incidental case of an occult breast carcinoma micrometastasis in papillary thyroid carcinoma: A view within a view. Thyroid Res Pract. 2018;15(3):142-146.

21. Sharma S, Singh M, Bhuyan G, et al. Extragonadal dysgerminoma presenting as neck metastasis and masquerading as a thyroid swelling. Clin Cancer Investig J. 2016;5(1):43-45.

22. Sharma S, Rana BP. Giant fibroadenoma of breast: a diagnostic dilemma in a middle aged woman. Adv Cytol Pathol. 2017;2(4):109-112.

23. Sharma S, Ahluwalia C, Singh M, et al. Diagnostic utility and efficacy of conventional versus surepath ${ }^{\circledR}$ liquid-based cytology in head and neck pathology: A study in an Indian tertiary care hospital. Iran J Pathol. 2018;13(2):188-195.

24. Sharma S, Sharma A. Pleomorphic adenoma of the buccal mucosa: a rare entity. J Cancer Prev Curr Res. 2019;10(6):146-149.

25. Sharma S, Kolte S. Gouty tophi - diagnosis clinched on cytology with review of the literature. Rec Adv Path Lab Med. 2015;1(2):13-16.

26. Dubb M, Michelow P. Cytologic features of chondroid syringoma in fine needle aspiration biopsies: a report of 3 cases. Acta Cytol. 2010;54(2):183-186.

27. Mishra K, Agarwal S. Fine needle aspiration cytology of malignant chondroid syringoma: a case report. Acta Cytol. 1998;42(5):1155-1158.

28. Obaidat NA, Alsaad KO, Ghazarian D. Skin adnexal neoplasms-part 2: An approach to tumours of cutaneous sweat glands. J Clin Pathol. 2007;60(2):145-159.

29. Malik R, Saxena A, Kamath N. A rare case of malignant chondroid syringoma of scalp. Indian Dermatol Online J. 2013;4(3):236--238.

30. Ishimura E, Iwamoto H, Kobashi Y, et al. Malignant chondroid syringoma: report of a case with widespread metastasis and review of pertinent literature. Cancer. 1983;52(10):1966-1973.

31. Mare GD, Vassallo L, Voglino C, et al. Chondroid syringoma: report of a case with uncommon location. J Med Diagn Meth. 2014;3:153.

32. Chen AH, Moreano EH, Houston B, et al. Chondroid syringoma of the head and neck: clinical management and literature review. Ear Nose Throat J. 1996;75(2):104-108.

33. Barnett MD, Wallack MK, Zuretti A, et al. Recurrent malignan chondroid syringoma of the foot: a case report and review of the literature. Am J Clin Oncol. 2000;23(3):227-232.

34. Anderson PJ, Rahilly MA. Chondroid syringoma of the foot. The Foot 1994;4(2):113-116.

35. Madi K, Attanasio A, Cecunjanin F, et al. Chondroid syringoma of the foot: a rare diagnosis. J Foot Ankle Surg. 2016;55(2):373-378.

36. Sundling RA, So E, Logan DB. Chondroid syringoma. Foot Ankle Spec. 2017;10(2):167-169. 